\title{
The Problem of Pawns
}

\author{
Tricia Muldoon Brown \\ Department of Mathematical Sciences \\ Georgia Southern University \\ Savannah, Georgia, U.S.A. \\ tmbrown@georgiasouthern. edu
}

Submitted: Nov 13, 2018; Accepted: Jul 12, 2019; Published: Aug 2, 2019

(C) The author. Released under the CC BY-ND license (International 4.0).

\begin{abstract}
Using a bijective proof, we show the number of ways to arrange a maximum number of nonattacking pawns on a $2 m \times 2 m$ chessboard is $\left(\begin{array}{c}2 m \\ m\end{array}\right)^{2}$, and more generally, the number of ways to arrange a maximum number of nonattacking pawns on a $2 n \times 2 m$ chessboard is $\left(\begin{array}{c}m+n \\ n\end{array}\right)^{2}$.
\end{abstract}

Mathematics Subject Classifications: 05A10, 05A19

\section{Introduction}

A set of pieces on a chessboard is said to be independent if no piece may attack another. Independence problems on chessboards have long been studied; both in terms of the size of a maximum arrangement as well as the number of such arrangements. For all traditional chess pieces, kings, queens, bishops, rooks, knights, and pawns, the maximum size of an independent set is known. While many of these counts have been recognized for years, books such as those by Dudeney [2], Kraitchik [4], Madachy [6], Watkins [9], and Yaglom and Yaglom [10] provide good resources for discussions of these results.

When considering the problem of counting the number of maximum arrangements, some of the pieces, for example rooks and bishops, have elementary solutions. Another type of piece utilizes a different kind of chessboard mathematics, that is, showing there is only one or two maximum arrangements of knights uses results on knight's tours. In the case of queens, the number of maximum independent arrangements is unknown in general, with the most recent count for $n=27$ generated through long-running computations, see Prueßer and Engelhardt [8]. For kings an asymptotic approximation is given by Larson [5], but an exact value is also unknown for even-length chessboards. Table 1 illustrates the known enumerative results including those shown in this paper, that is, here we wish to enumerate the number of maximum arrangements of nonattacking pawns. Arrangements of nonattacking pawns have been studied by Kitaev and Mansour [3] who provide upper 


\begin{tabular}{ccccccc}
\hline & Kings & Queens & Bishops & Knights & Rooks & Pawns \\
\hline $\begin{array}{c}\text { Number of pieces } \\
\text { in a maximum } \\
\text { arrangement }\end{array}$ & $\left\lceil\frac{n}{2}\right\rceil^{2}$ & $n$ & $2 n-2$ & $\left\lceil\frac{n}{2}\right\rceil^{2}+\left\lfloor\frac{n}{2}\right\rfloor^{2}$ & $n$ & $n\left\lceil\frac{n}{2}\right\rceil$ \\
\hline $\begin{array}{c}\text { Number of } \\
\text { maximum } \\
\text { arrangements }\end{array}$ & $\begin{array}{c}1 \text { if } n \text { is odd, } \\
\text { unknown if } \\
n>26 \text { is even }\end{array}$ & $\begin{array}{c}\text { unknown if } \\
n>27\end{array}$ & $2^{n}$ & $\begin{array}{c}1 \text { if } n \text { is odd, } \\
2 \text { if } n \text { is even }\end{array}$ & $n !$ & $\begin{array}{c}\left(\begin{array}{c}n \\
n / 2\end{array}\right)^{2} \text { if } n \text { is odd, } \\
\text { is even }\end{array}$ \\
\hline
\end{tabular}

Table 1: Enumerative results for maximum independent sets placed on an $n \times n$ chessboard where $n>1$

and lower bounds on the number of all, not just maximum, arrangements of pawns on $2 m \times 2 n$ rectangles in terms of Fibonacci numbers.

As there are only two distinct arrangements for odd length chessboards, we focus on boards with even length. Because we can divide a $2 m \times 2 m$ chessboard into $m^{2} 2 \times 2$ squares each with at most two pawns, the maximum number of independent pawns is at most $2 m^{2}$. This value is easily achieved, and examples are illustrated in Figure 1.
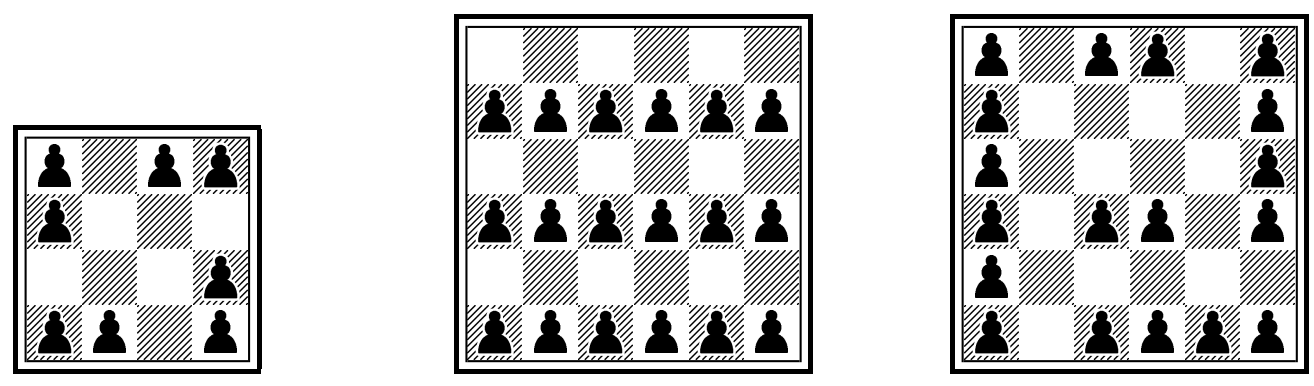

Figure 1: Arrangements of nonattacking pawns for even length chessboards

We will provide a bijection between the set of maximum nonattacking arrangements of pawns on a $2 m \times 2 m$ chessboard and the set of subsets of $m$ rows and $m$ columns of a $2 m \times 2 m$ matrix.

\section{A bijection between arrangements of nonattacking pawns and pairs of subsets}

Instead of considering full arrangements of nonattacking pawns on a $2 m \times 2 m$ chessboard, we first consider arrangements on a $2 \times 2$ chessboard. There are four possible arrangements labeled with $\mathrm{A}, \mathrm{B}, \mathrm{C}$, and $\mathrm{D}$, as illustrated in Figure 2. We define a function $f$ on this set, where $f(A)=D$ and $f(B)=f(C)=f(D)=C$. We use this function to define an $(m+1) \times(m+1)$ matrix $M_{2 m}=\left(m_{i, j}\right)_{1 \leqslant i, j \leqslant m+1}$ whose entries correspond to arrangements of $2 m$ independent pawns on a $2 \times 2 m$ rectangular chessboard. 


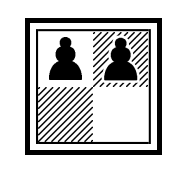

(a) Type A

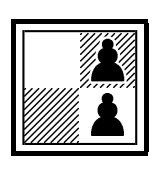

(b) Type B

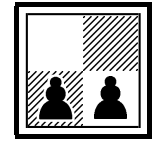

(c) Type C

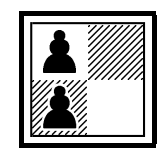

(d) Type D

Figure 2: The four maximum arrangements of 2 pawns on a $2 \times 2$ chessboard

Definition 1. Let $M_{2 m}=\left(m_{i, j}\right)_{1 \leqslant i, j \leqslant m+1}$ be the matrix whose entries consist of arrangements of $2 m$ nonattacking pawns on a $2 \times 2 m$ rectangular chessboard. We can think of each rectangle as a string of $m 2 \times 2$ squares, each with exactly two pawns. The entries of $M_{2 m}$ are defined as follows:

i. For $1 \leqslant j \leqslant m+1$, let $m_{1, j}$ be the arrangement where the leftmost $(m+1-j) 2 \times 2$ squares of the rectangular chessboard are of Type A and the remaining rightmost $(j-1)$ squares are of Type B.

ii. For $1 \leqslant i \leqslant m+1$, use $m_{1, j}$ to generate the arrangements $m_{i, j}$ by replacing the leftmost $(i-1) 2 \times 2$ squares of $m_{1, j}$, with their image under the function $f$ and leaving the rightmost $(m+1-i) 2 \times 2$ squares fixed.

See Figure 3 for an example of an entry in the first row and fifth row of $M_{14}$, and see Figure 4 for the entire matrix $M_{6}$. We claim this matrix contains all possible nonattacking arrangements of pawns on a $2 \times 2 m$ rectangular chessboard.

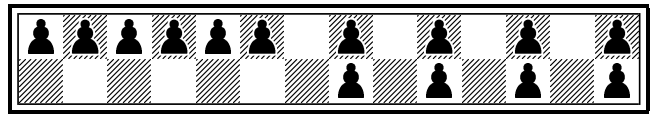

(a) Entry $m_{1,5}$ in the matrix $M_{14}$

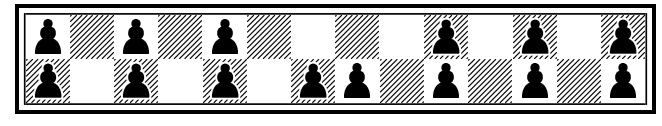

(b) Entry $m_{5,5}$ in the matrix $M_{14}$

Figure 3: Entries from the matrix $M_{14}$

Proposition 2. Every nonattacking arrangement of $2 m$ pawns on a $2 \times 2 m$ rectangle appears exactly once in the matrix $M_{2 m}$.

Proof. To begin, we show the number of distinct arrangements of pawns on a $2 \times 2 \mathrm{~m}$ rectangle is $(m+1)^{2}$. For $m=1$, a $2 \times 2$ square has the four distinct arrangements shown in Figure 2, so we induct on $m$. The leftmost $2 \times 2$ square of a $2 \times 2 m$ rectangle may have Type A, B, C, or D. First, assume this leftmost square has Type D. Any maximum independent arrangement of a $2 \times 2(m-1)$ rectangle may be appended to the Type $\mathrm{D}$ square creating $m^{2}$ distinct maximum nonattacking arrangements. Next, if the leftmost 

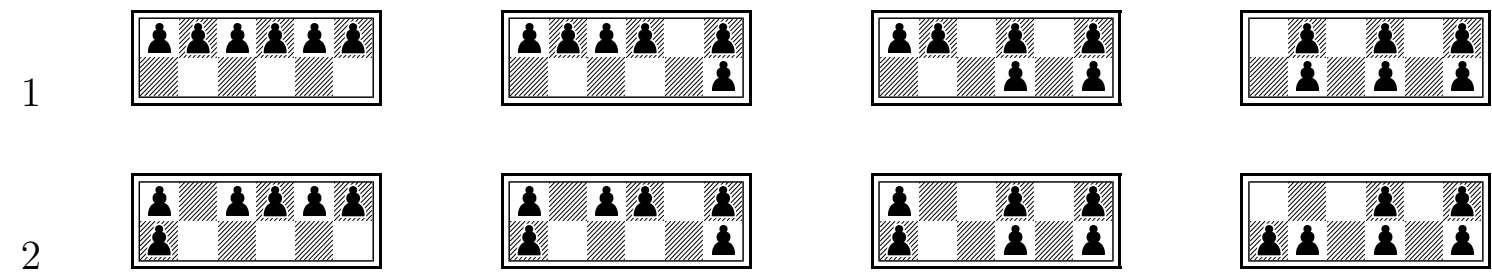

\section{2}
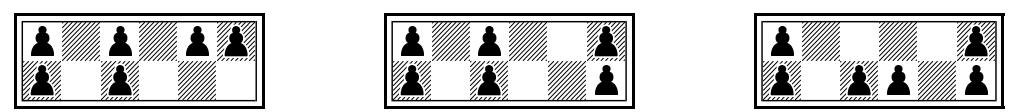

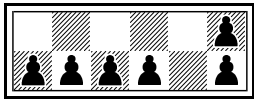
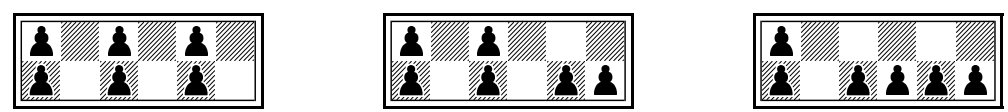

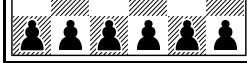

Figure 4: Entries in the matrix $M_{6}=\left(m_{i, j}\right)_{1 \leqslant i, j \leqslant 4}$

square has Type A or C, it must be followed by a square of same type or of Type B. But in any $2 \times 2 m$ rectangle, when reading from left to right, as soon as a Type B square is introduced in the strip, all remaining squares to the right must also be of Type B. Thus any $2 \times 2 m$ strip beginning with a Type A or Type $\mathrm{C}$ square consists of $k$ squares of Type A or $\mathrm{C}$ followed by $m-k$ squares of Type $\mathrm{B}$ for $1 \leqslant k \leqslant m$. Finally there is one possible arrangement beginning with a Type B square. Thus we have

$$
m^{2}+2 m+1=(m+1)^{2}
$$

distinct arrangements as desired.

Further, no arrangement appears more than once in the matrix $M_{2 m}$. We continue to think of the entries of the matrix $M_{2 m}$ as a string of $m 2 \times 2$ squares. We observe, by construction, as one reads from top to bottom down a column of the matrix, the only actions on these $2 \times 2$ squares are:

i. Type A squares may be changed to Type D squares.

ii. Type B squares may be changed to Type C squares.

iii. Any type square may remain fixed.

Similarly, as you read from left to right across a row of the matrix, the only actions are:

i. Type A squares may be changed to Type B squares.

ii. Type D squares may be changed to Type C squares.

iii. Any type square may remain fixed. 
Given any two arrangements in distinct positions in the matrix $M_{2 m}$, at least one square has changed from the lower-indexed entry to the higher-indexed entry. If that square was of Type B or D, respectively, it was changed into a Type $\mathrm{C}$ square and no action may change it back to a Type B or D square, respectively. If the square was of Type A, then it was changed to a Type B, C, or D square, but in any case, may not return to Type A. Because Type $\mathrm{C}$ squares cannot be changed, we have a matrix with unique elements whose size is equal to the size of the set, so therefore each independent maximum arrangement of pawns occurs exactly once in $M_{2 m}$.

Now, we define a map from the set of subsets of $m$ rows and $m$ columns of a $2 m \times 2 m$ matrix into the set of nonattacking arrangements of $2 m^{2}$ pawns.

Definition 3. Suppose the rows and columns of a $2 m \times 2 m$ matrix are indexed by $[2 m]$. Set

$$
A=\{C \cup R: C, R \subset[2 m] \text { and }|C|=|R|=m\},
$$

that is, $A$ is the set of all subsets consisting of $m$ rows $R=\left\{r_{1}, r_{2}, \ldots, r_{m}\right\} \subset[2 m]$ and $m$ columns $C=\left\{c_{1}, c_{2}, \ldots, c_{m}\right\} \subset[2 m]$. Let $B$ be the set of all nonattacking arrangements of $2 m^{2}$ pawns on a $2 m \times 2 m$ chessboard. Define the map $\Phi: A \longrightarrow B$ as follows:

Given a subset $C \cup R$, assume without loss of generality that $r_{1}<r_{2}<\cdots<r_{m}$ and $c_{1}<c_{2}<\cdots<c_{m}$. Then set $S$ to be the set of $m$ ordered pairs where

$$
S=\left\{\left(a_{i}, b_{i}\right):\left(a_{i}, b_{i}\right)=\left(r_{i}-i+1, c_{i}-i+1\right) \text { for } 1 \leqslant i \leqslant m\right\} .
$$

For each ordered pair $\left(a_{i}, b_{i}\right)$, identify the $2 \times 2 m$ chessboard arrangement $m_{a_{i}, b_{i}}$ from the matrix $M_{2 m}$. Concatenate these strips sequentially so that $m_{a_{i}, b_{i}}$ is directly above $m_{a_{i+1}, b_{i+1}}$ for $1 \leqslant i \leqslant m-1$ to create an arrangement of $2 m^{2}$ pawns on a $2 m \times 2 m$ chessboard. This arrangement is the image of the subset $C \cup R$ under $\Phi$.

Example 4. Given $2 m=6$, suppose $R=\{1,4,5\}$ and $C=\{2,4,6\}$. Then $S=$ $\{(1,2),(3,3),(3,4)\}$. Thus, we concatenate the arrangements $m_{1,2}, m_{3,3}, m_{3,4}$ from Figure 4 to get the maximum $6 \times 6$ arrangement:

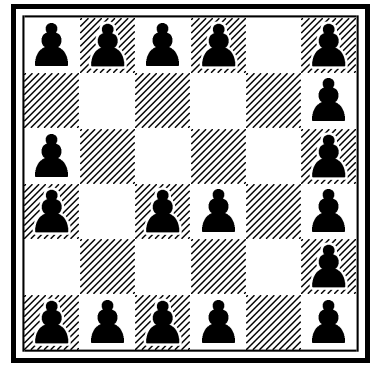

Example 5. Given $2 m=8$, suppose $R=\{2,3,4,8\}$ and $C=\{1,6,7,8\}$. Then $S=$ $\{(2,1),(2,5),(2,5),(5,5)\}$ and we have the following $8 \times 8$ arrangement: 


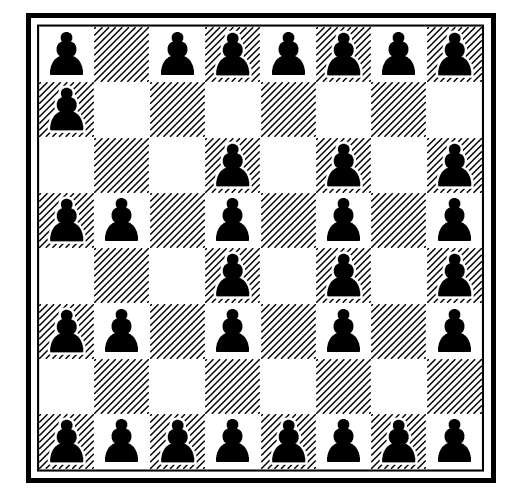

We check that the arrangements of pawns given by the function $\Phi$ are nonattacking.

Proposition 6. Each arrangement of $2 m^{2}$ pawns on a $2 m \times 2 m$ chessboard in the image $\Phi(A)$ is independent.

Proof. By construction, we know the the pawns may not attack within each $2 \times 2 m$ rectangular chessboard, so it is left to show that the pawns may not attack from one rectangle to another. We apply the restrictions on movement along rows and columns noted in the proof of Proposition 2.

Let $a=m_{i, j}$ and $b=m_{i^{\prime}, j^{\prime}}$ be any two nonattacking arrangements from the matrix $M_{2 m}$ such that $i \leqslant i^{\prime}$ and $j \leqslant j^{\prime}$. We assume $a$ lies directly above $b$ in a maximum arrangement of independent pawns on the $2 m \times 2 m$ chessboard. Divide each $2 \times 2 m$ rectangle into $2 \times 2$ squares and denote a $2 \times 2$ square of $a$, or $b$ respectively, at position $k$ where $1 \leqslant k \leqslant m$ by $A_{k}$ or $B_{k}$, respectively. First, if $A_{k}$ has Type A, then the pawns in $A_{k}$ may not attack any pawns in the arrangement $b$. Next, suppose $A_{k}$ has Type $\mathrm{B}$, so thus $B_{k}$ has Type B or Type C. In either case the pawns in $A_{k}$ may not attack the pawns in $B_{k}$. However the pawn in $A_{k}$ may also attack the upper left corner of $B_{k+1}$. Because the square $A_{k+1}$ must also have Type B, we know $B_{k+1}$ has Type B or C. In either case there is no pawn in the upper left corner, so pawns in $A_{k}$ may not attack pawns in $B_{k+1}$. Similarly, if $A_{k}$ has Type $\mathrm{D}$ then $B_{i}$ also has Type $\mathrm{D}$, and thus no attack is possible. In this case a pawn in $A_{i}$ could also attack the upper right corner of $B_{i-1}$. We see that $A_{i-1}$ also has Type $\mathrm{D}$, so $B_{k-1}$ has Type $\mathrm{D}$ and thus no attack is possible from $A_{k}$ to $B_{k-1}$. Finally, suppose $A_{k}$ is of Type $\mathrm{C}$, so pawns in $A_{k}$ may attack squares $B_{k-1}, B_{k}$, and $B_{k+1}$. We know $A_{k-1}$ is of Type $\mathrm{C}$ or Type $\mathrm{D}$ and $A_{k+1}$ is of Type C. So we have that $B_{k-1}$ is of Type $\mathrm{C}$ or $\mathrm{D}$, thus not susceptible to an attack from $A_{k}$. The squares $B_{k}$ and $B_{k+1}$ are both of Type $\mathrm{C}$ and also have no pawns that may be attacked by pawns in $A_{k}$. Finally, we note in any case, if the squares $B_{k-1}$ or $B_{k+1}$ do not exist, then trivially there is no attacking pawn. Therefore, we have shown that any entry weakly to the left or above another entry in $M_{2 m}$ may not attack when placed directly above the second entry, and thus have proven the claim.

We have shown that each subset in $A$ provides exactly one maximum nonattacking arrangement of pawns on a $2 m \times 2 m$ chessboard, thus $\Phi(A) \subseteq B$. It is left to show that no other maximum independent arrangements are possible. 
Proposition 7. Every nonattacking arrangement of $2 m^{2}$ pawns on a $2 m \times 2 m$ chessboard is the image of a subset $C \cup R \in A$ under the map $\Phi$.

Proof. Any arrangement of $2 m^{2}$ pawns on a $2 m \times 2 m$ chessboard may be divided into $m 2 \times 2 m$ rectangular boards which correspond to the entries $\left(m_{a_{1}, b_{1}}, \ldots, m_{a_{m}, b_{m}}\right)$ in the matrix $M_{2 m}$. For all $i$, as long as $a_{i} \leqslant a_{i+1}$ and $b_{i} \leqslant b_{i+1}$, then the arrangement is an element of the image $\Phi(A)$. Suppose to the contrary $a_{i}>a_{i+1}$ for some $i$. This implies the arrangement $m_{a_{i}, b_{i}}$ is in a lower row in $M_{2 m}$ than arrangement $m_{a_{i+1}, b_{i+1}}$, but appears directly above $m_{a_{i+1}, b_{i+1}}$ in the $2 m \times 2 m$ arrangement. We apply a similar argument to that used in Proposition 6. At least one square, say $A_{k}$ in $m_{a_{i}, b_{i}}$ is different from the square in the same position, $B_{k}$, in $m_{a_{i+1}, b_{i+1}}$. If $A_{k}$ is of type $\mathrm{D}$, then $B_{k}$ is of type $\mathrm{A}$ or $\mathrm{C}$, hence the pawn in the lower left corner of $A_{k}$ may attack the pawn in the upper right corner of $B_{k}$. If $A_{k}$ is of Type $\mathrm{C}$, then $B_{k}$ is of Type $\mathrm{A}$ or $\mathrm{B}$, and the pawn in the lower left corner of $A_{k}$ may attack the pawn in the upper right corner of $B_{k}$. Thus $a_{i} \ngtr a_{i+1}$. Similarly, if $b_{i}>b_{i+1}$, the arrangement $m_{a_{i}, b_{i}}$ is in column further to the right in $M_{2 m}$ than arrangement $m_{a_{i+1}, b_{i+1}}$, but appears directly above $m_{a_{i+1}, b_{i+1}}$ in the $2 m \times 2 m$ arrangement. Again at least one square, say $A_{k}$ in $m_{a_{i}, b_{i}}$ is different from the square in the same position, $B_{k}$, in $m_{a_{i+1}, b_{i+1}}$. If $A_{k}$ of Type B, then $B_{k}$ is of Type A or D and the pawn in the lower right corner of $A_{k}$ may attack the pawn in the upper left corner of $B_{k}$. Further if $A_{k}$ is of Type $\mathrm{C}$, then $B_{k}$ is of Type A or D and the pawn in the lower right corner of $A_{k}$ may attack the pawn in the upper left corner of $B_{k}$. Thus $b_{i} \ngtr b_{i+1}$, and we have arrived at the contradiction.

Therefore we have the following corollary.

Corollary 8. The function $\Phi: A \longrightarrow B$ is a bijection. result.

Hence, because we may choose an $m$-subset of $[2 m]$ in $\left(\begin{array}{c}2 m \\ m\end{array}\right)$ ways, we have our main

Theorem 9. The number of maximum nonattacking arrangements of pawns on a $2 m \times 2 m$ chessboard is $\left(\begin{array}{c}2 m \\ m\end{array}\right)^{2}$.

We may generalized this result to maximum independent arrangements of pawns on $2 n \times 2 m$ rectangles.

Theorem 10. The number of maximum nonattacking arrangements of pawns on a $2 n \times$ $2 m$ chessboard is $\left(\begin{array}{c}m+n \\ n\end{array}\right)^{2}$.

Proof. Assume without loss of generality that $n \leqslant m$. We may utilize the bijection $\Phi$ from above. Given a nonattacking arrangement of $2 m n$ pawns on a $2 n \times 2 m$ chessboard, we may divide the arrangement into $n$ rectangles of size $2 \times 2 \mathrm{~m}$. These correspond to $n$ (not necessarily distinct) entries in the matrix $M_{2 m}$. Thus we have a set of indices from the matrix entries

$$
S=\left\{\left(a_{i}, b_{i}\right) \mid 1 \leqslant a_{1} \leqslant a_{2} \leqslant \cdots \leqslant a_{n} \leqslant m+1 \text { and } 1 \leqslant b_{1} \leqslant b_{2} \leqslant \cdots \leqslant b_{n} \leqslant m+1\right\} .
$$


Two create distinct column and row entries we have

$$
C \cup R=\left\{a_{1}, a_{2}+1, a_{3}+2, \ldots, a_{n}+n-1\right\} \cup\left\{b_{1}, b_{2}+1, b_{2}+3, \ldots, b_{n}+n-1\right\} .
$$

We note the maximum value of elements in $C$ or $R$ is $m+n$, thus $C, R \subset[m+n]$. Hence we are choosing an $n$-subset of rows from $[m+n]$ and an $n$-subset of columns from $[m+n]$, and the result follows.

\section{Connections with lattice paths and directed polyominoes}

There are other combinatorial objects enumerated by $\left(\begin{array}{c}2 m \\ m\end{array}\right)^{2}$. Entry A002894 in the Online Encyclopedia of Integer Sequences [7] provides an overview of some of these objects. Here we want to examine two types of lattice paths and a set of directed polyominoes. The first type of lattice path is a path on the integer lattice from the point $(0,0)$ to $(2 m, 2 m)$ using only north and east steps and passing through the point $(m, m)$. Let $L$ be the set of all such lattice paths. It is not hard to see that $L$ is in bijection with $A$, that is, the set of pairs of subsets $(C, R)$ where $C, R \subset[2 m]$ and $|C|=|R|=m$. Note any lattice path from $(0,0)$ to $(m, m)$ has exactly $2 m$ steps of which $m$ are east steps and $m$ are north steps. To define the path from $(0,0)$ to $(m, m)$ simply let $C$ indicate the positions of the east steps among the $2 m$ total steps, and further let $R$ indicate the positions of the $m$ north steps among the $2 m$ total steps between $(m, m)$ and $(2 m, 2 m)$. Because the set of lattice paths is in bijection with $A$, composing with the map $\Phi$ gives a bijection between the set of lattice paths $L$ and the set of maximum arrangements of nonattacking pawns on a $2 m \times 2 m$ chessboard.

Another interesting combinatorial object is a directed-convex polyomino. A polyomino is finite union of cells on a square lattice with a simply connected interior. The polyomino is convex if the intersection of each row and each column with a horizontal, respectively vertical, line of cells is connected. Finally, the convex polyomino is directed if each cell may be reached from a distinguished cell, called the root, using only north and east steps. Barcucci, Frosini, and Rinaldi [1] provide a bijection between the set of directed polyominoes contained in an square with side length $m+1$ and a subset of 2-colored grand Motzkin paths. In this case, a 2-colored grand Motzkin path is a path from $(0,0)$ to $(0,2 m)$ using up steps of the form $(1,1)$, down steps of the form $(1,-1)$, and two colors of horizontal steps of the form $(1,0)$. Here we use the specific condition that the number of horizontal steps of each color must be equal, although Barcucci, Frosini, and Rinaldi prove a more general bijection by removing this condition and allowing directed polyominoes in a rectangle. These authors do not provide a bijection directly from the set of polyominoes into the set of 2-colored grand Motzkin paths, but instead utilize a set of $2 \times 2 m$ matrices whose entries are either 0 or 1 . Our condition states then that each row has an equal number of 0's and 1's. Of course this means that each row has exactly $m$ 0's and $m$ 1's, so thus we have a simple bijection from $A$ to the set of such matrices by letting $C$ and $R$ indicate the positions of the $m$ 1's in the upper and lower rows of the matrix, respectively. Further, by composing $\Phi$ with this bijection and the bijections 
by Barcucci, Frosini, and Rinaldi, we have a map between nonattacking arrangements of pawns on a $2 m \times 2 m$ chessboard and 2-colored grand Motzkin paths of length $2 m$ having an equal number of horizontal steps of each color as well as between the arrangements of pawns and directed polyominoes contained in a $(m+1) \times(m+1)$ square.

\section{References}

[1] E. Barcucci, A. Frosini, and S. Rinaldi. On directed-convex polyominoes in a rectangle. Discrete Math., 298(1-3):62-78, 2005.

[2] H. E. Dudeney. Amusements in Mathematics. Thomas Nelson \& Sons, Limited, Edinburgh, 1917.

[3] S. Kitaev and T. Mansour. The problem of the pawns. Ann. Comb., 8(1):81-91, 2004.

[4] M. Kraitchik. Mathematical Recreations. W. W. Norton \& Company Inc, New York, 1942.

[5] M. Larsen. The problem of kings. Electron. J. Combin., 2:\#R18, 1995.

[6] J. S. Madachy. Madachy's Mathematical Recreations. Dover, New York, 1979.

[7] N. J. A. Sloane. editor, The On-Line Encyclopedia of Integer Sequences. Published electronically at https://oeis.org, 2019.

[8] T. B. Preußer and M. R. Engelhardt. Putting Queens in Carry Chains, No. 27. J. Signal Process. Syst., 88(2):185-201, 2017.

[9] J. J. Watkins. Across the Board: The Mathematics of Chessboard Problems. Princeton University Press, Princeton, 2004.

[10] A. M. Yaglom and I. M. Yaglom. Challenging Mathematical Problems with Elementary Solutions. Vol I: Combinatorial Analysis and Probability Theory. Dover, New York, 1987. 